Palavras chave: Cupins subterrâneos Durabilidade natural Ensaio biológico

Histórico: Recebido 22/07/2014 Aceito 21/09/2015

Keywords: Subterranean termites Natural durability Biological assay

Correspondência: jbp2@uol.com.br

$\mathrm{DOI}$
Juarez Benigno Paes', Victor Fassina Brocco', Jordão Cabral Moulin², Javan Pereira Motta Rejane Costa Alves ${ }^{4}$

\section{EFEITOS DOS EXTRATIVOS E DA DENSIDADE NA RESISTÊNCIA NATURAL DE MADEIRAS AO TÉRMITA Nasutitermes corniger}

RESUMO: A avaliação da resistência natural da madeira a organismos xilófagos é de importância fundamental na escolha de espécies a serem utilizadas na construção civil e na indústria de móveis. Assim, avaliaram-se os efeitos do teor de extrativos e da densidade na resistência biológica das madeiras de Acacia mangium, Casuarina equisetifolia, Corymbia torelliana, Eucalyptus cloeziana, Tectona grandis e Caesalpinia echinata ao térmita xilófago Nasutitermes corniger, em condições de laboratório. Corpos de prova, com dimensões de $2,00 \times 2,54 \times 0,64 \mathrm{~cm}$ (radial $\times$ longitudinal $\times$ tangencial) foram retirados de cada madeira em quatro posições no sentido medula-casca (cerne interno, cerne mediano, cerne externo e alburno). As madeiras foram expostas à ação dos térmitas por 28 dias em ensaio de alimentação forçada. As amostras não selecionadas para o ensaio com os térmitas foram transformadas em serragem e os extrativos obtidos da fração que passou pela peneira de 40 e ficou retida na de 60 "mesh". A resistência natural da madeira, dentre as posições medula-casca, para as espécies estudadas, não está correlacionada com a densidade e teor de extrativos das mesmas. No entanto, entre as madeiras, aquelas com maiores densidade e teor de extrativos são mais resistentes. As madeiras com maior resistência biológica ao térmita Nasutitermes corniger (menores perda de massa, desgaste e tempo de sobrevivência dos insetos) são Corymbia torelliana e Caesalpinia echinata e a de menor resistência a Casuarina equisetifolia.

\section{EFFECTS OF EXTRACTIVES AND DENSITY ON NATURAL RESISTANCE OF WOODS TO TERMITE Nasutitermes corniger}

ABSTRACT: The evaluation of the natural resistance of wood to wood-destroying organisms is of fundamental importance in the choice of species to be used in buildings and furniture industry. Thus, the effects of extractives and wood density on biological resistance of Acacia mangium, Casuarina equisetifolia, Corymbia torelliana, Eucalyptus cloeziana, Tectona grandis and Caesalpinia echinata woods to the xylophagous termite Nasutitermes corniger was evaluated under laboratory conditions. Test samples, with dimensions of $2.00 \times 2.54 \times 0.64 \mathrm{~cm}$ (radial $\times$ tangential $\times$ longitudinal) in four positions in pith-bark direction (internal heart, intermediate heart, outer heart and sapwood) were taken. The woods were exposed to termite action for 28 days in no-choice feeding test. The samples not selected for the termite test were turned into sawdust and the extractive contents were obtained using the shavings that passed through the sieve of 40 and were retained in the sieve of 60 mesh. The wood natural resistance, within the pithbark positions, for the studied species, is not correlated with the density and extractive content. However, among the woods, those with higher density and extractive content are more resistant. The woods with greater biological resistance to the termite Nasutitermes corniger (smaller mass loss, waste and survival time of insects) are Corymbia torelliana and Caesalpinia echinata and of less resistance is Casuarina equisetifolia.

\footnotetext{
' Universidade Federal do Espírito Santo - Jerônimo Monteiros, Espírito Santo, Brasil

2 Universidade Federal de Lavras - Lavras, Minas Gerais, Brasil

3 Universidade do Estado do Pará - Marabá, Pará, Brasil

${ }^{4}$ Universidade Federal Rural do Semi-Árido - Mossoró, Rio Grande do Norte, Brasil
} 


\section{INTRODUÇÃO}

A madeira está sujeita a deterioração, - que limita o seu uso na construção civil e na indústria de móveis. A resistência à deterioração pode ser determinada pela exposição da madeira a agentes bióticos ou abióticos (PAES et al., 20I3). Dentre os bióticos, os fungos apodrecedores e os térmitas (Isoptera) são os responsáveis pela maior deterioração (TREVISAN, 2006).

Os térmitas de hábitos subterrâneos são os de maior importância econômica pelos danos causados à madeira (PAES et al.,2003; GONÇALVES; OLIVEIRA, 2006, PAES et al., 2013). Espécies do gênero Nasutitermes atacam móveis e principalmente, madeiras utilizadas nas estruturas das construções, nos meios rural e urbano (PAES et al., 2003), sendo a espécie Nasutitermes corniger de ocorrência frequente em várias regiões brasileiras.

Desta forma estudos que avaliem a resistência biológica da madeira de espécies florestais com potencial de uso para construção civil e setor moveleiro são relevantes para o setor (SILVA et al. 2004; RUDI et al. 2012), a fim de otimizar o uso e evitar gastos desnecessários com a reposição prematura de peças deterioradas (PAES et al., 2009).

A acácia (Acacia mangium) é uma espécie florestal de rápido crescimento com boa capacidade de adaptação às condições edafoclimáticas brasileiras. Sua madeira possui múltiplos usos, como em produtos serrados para a indústria de móveis e construção civil (BALIEIRO et al., 2004). A casuarina (Casuarina equisetifolia) também possui facilidade de adaptação às condições brasileira, cuja madeira e utilizada em construções diversas (SILVA et al., 2008).

Espécies de eucalipto são plantadas no Brasil com diversas finalidades de usos. A madeira de Corymbia torelliana tem boa estabilidade dimensional podendo ser utilizada na produção de madeira serrada, em decorrência de poucos defeitos como arqueamento, encurvamento e rachaduras de topo (HORNBURG et al., 2012). O Eucalyptus cloeziana tem boa qualidade para a produção de madeira serrada, fabricação de pisos e uso na construção civil (ALMEIDA, 2006).

A teca (Tectona grandis) vem sendo plantada em várias regiões no Brasil e sua madeira utilizada para serraria, laminados e compensados (MOTTA et al., 20I3). O pau-brasil (Caesalpinia echinata) é uma espécie mundialmente utilizada para a confecção de $\operatorname{arcos}$ e instrumentos de corda, por causa de suas características de ressonância, estabilidade dimensional, beleza e durabilidade (ANGYALOSSY et al., 2005, MARQUES et al., 20I2).

A resistência biológica da madeira pode variar com a espécie e a posição no lenho, e, na maioria das vezes, está ligada à quantidade e tipo de extrativos presentes (FINDLAY, 1985). Os extrativos são formados durante a transformação do alburno em cerne, sendo a parte externa, região fronteiriça com o alburno, mais resistente que a porção interna, formada quando a planta é jovem. Porém, essas variações não ocorrem para todas as espécies (PANSHIN; DE ZEEUW, I980; FINDLAY, 1985).

Assim, avaliaram-se os efeitos dos extrativos e da densidade na resistência natural das madeiras de seis espécies florestais, ao ataque do térmita xilófago Nasutitermes corniger, em condições de laboratório.

\section{MATERIAL E MÉTODOS}

\section{Espécies utilizadas e confecção das amostras}

Foram utilizadas madeiras das espécies exóticas Acacia mangium, Casuarina equisetifolia, Corymbia torelliana, Eucalyptus cloeziana, Tectona grandis, além da madeira de Caesalpinia echinata. As madeiras foram obtidas no Laboratório de Usinagem e Beneficiamento da Madeira (LUMber), Departamento de Ciências Florestais e da Madeira (DCFM), Centro de Ciências Agrárias (CCA), Universidade Federal do Espírito Santo (UFES), no Município de Jerônimo Monteiro, Espírito Santo, Brasil.

Peças radiais (tábuas) com o cerne e 0 alburno intactos foram selecionadas de cada espécie florestal. Uma secção isenta de defeitos, como manchas de fungos ou rachaduras, foi retirada de tábuas, proveniente da primeira tora da árvore, região próxima a $1,30 \mathrm{~m}$ do solo, de aproximadamente 50 $\mathrm{cm}$ de comprimento. A secção foi subdividida em oito partes radiais, diametralmente opostas e de mesma dimensão, que foram agrupadas duas a duas e identificadas conforme sua posição em relação à medula (cerne interno, cerne mediano, cerne externo e alburno). Assim, toda a madeira foi representada (PAES et al., 2009; ALENCAR et al., 201 I).

As amostras de madeira foram aplainadas para 2,00 cm, no sentido radial, para homogeneizar as dimensões. Posteriormente, foram transformadas em corpos de prova de 2,54 ×2,00 x 0,64 cm (longitudinal $x$ radial $x$ tangencial). Oito amostras, isentas de defeitos, 
foram selecionadas para cada posição e espécie florestal, totalizando 192 amostras, as quais foram utilizadas no ensaio com cupins xilófagos.

\section{Resistência natural da madeira a cupins xilófagos}

O ensaio foi realizado conforme a American Society for Testing and Materials - ASTMD - 3345 (2005), modificado por Paes et al. (20I0). O experimento foi montado em frascos de $600 \mathrm{~mL}$ com $200 \mathrm{~g}$ de areia e a umidade corrigida para $75 \%$ da capacidade de retenção de água com $38 \mathrm{~mL}$ de água destilada. Em cada frasco, foram adicionados um corpo de prova e I $\pm 0,05 \mathrm{~g}$ do térmita do gênero Nasutitermes corniger (Motsch.). A fim de se verificar a quantidade de insetos, foram realizadas, durante as pesagens, 10 contagens dos mesmos, tendo se verificado que em I,00 g havia o equivalente a \pm 380 indivíduos, sendo $80 \%$ de operários.

Conforme indicações da ASTM D - 3345 (2005), além das espécies testadas, foram utilizados também amostras de Pinus sp., a fim de comparar o potencial de ataque dos térmitas testados com outras pesquisas em que essa madeira é empregada.

Os frascos foram levemente tampados após a adição dos térmitas, para evitar a fuga dos insetos e permitir a circulação de ar. Cinco repetições para cada espécie e posição amostrada na direção radial do tronco foram avaliadas. Para avaliar a perda de massa operacional, foram montados frascos com as mesmas condições, porém sem a adição dos térmitas, para isto foram utilizadas três repetições.

O volume e a massa de cada amostra foram determinados, como o recomendado pela ASTM D I4I3 (2005), e utilizados para o cálculo da densidade da madeira e da perda de massa causada aos corpos de prova submetidos aos ensaios (ASTM D - 2017, 2005). $\mathrm{O}$ ensaio permaneceu em sala climatizada $\left(28 \pm 2{ }^{\circ} \mathrm{C}\right.$ e $75 \pm 5 \%$ de umidade relativa) por 28 dias. A resistência natural da madeira foi avaliada em função da perda de massa corrigida pela perda de massa operacional (ASTM D - 2017, 2005), do desgaste provocado pelos cupins e do número de dias para a morte de todos os insetos em cada frasco (ASTM D - 3345, 2005), (Tabela I).

Tabela I Avaliação do desgaste da madeira submetida aos térmitas. Table I Evaluation of wood waste submitted to the termites.

\begin{tabular}{lc}
\hline Tipo de Desgaste & Notas \\
\hline Sadio, permitindo escarificações superficiais & 10 \\
Ataque superficial & 9 \\
Ataque moderado, havendo penetrações & 7 \\
Ataque intensivo & 4 \\
Falha, havendo ruptura dos corpos de prova & 0 \\
\hline
\end{tabular}

Fonte: Adaptado de ASTM D - 3345 (2005).
Para a avaliação da sobrevivência dos térmitas, a atividade dos mesmos, em cada frasco, era observada diariamente, sempre no mesmo horário, e quando todos os insetos morriam, o número de dias para a sobrevivência dos mesmos foi contabilizado.

\section{Quantificação dos extrativos}

As amostras não selecionadas para o ensaio com térmitas foram transformadas em cavacos com dimensões semelhantes a palitos de fósforos e, posteriormente em serragem em moinho do tipo Willey. A serragem foi classificada e utilizada a porção que passou pela peneira de 40 e ficou retida na de 60 "mesh". A serragem classificada foi mantida à temperatura 20 $\pm 2{ }^{\circ} \mathrm{C}$ e $65 \pm 5 \%$ de umidade relativa e destinada a quantificação do teor de extrativos presentes na mesma.

O teor de extrativos da madeira foi determinado com uso de uma solução de etanol:tolueno (2:I v/v), em um aparelho tipo Soxhlet (ASTM D - II07, 2005). As análises químicas para a determinação dos extrativos foram realizadas em duplicatas.

\section{Análise estatística dos dados}

A resistência da madeira ao térmita Nasutitermes corniger foi avaliada em laboratório em delineamento inteiramente casualizado com arranjo fatorial (seis $x$ quatro) em que foi avaliado o efeito da espécie florestal (seis níveis) e da posição na direção radial (quatro níveis).

A correlação de Pearson foi utilizada para se avaliar a influência da densidade e dos extrativos nas variáveis relacionadas ao ensaio de resistência biológica das madeiras ao térmita testado (perda de massa, desgaste e mortalidade).

Os dados de perda de massa (\%) e da mortalidade (\%) foram transformados em arcsen $\left[(x / 100)^{0,5}\right]$, de desgaste (nota) e do tempo (dias) em $(x+0,5)^{0,5}$ (STEEL; TORRIE 1980), para permitir a normalidade (teste de Lilliefors) e a homogeneidade das variâncias (teste de Cochran e Bartellet). Foram realizadas as análises de variância e as médias comparadas pelo teste de Scott-Knott ( $p \leq 0,05)$, para os fatores e interação detectados como significativos pelo teste de $\mathrm{F}(\mathrm{p} \leq 0,05)$.

\section{RESULTADOS E DISCUSSÃO}

A densidade das madeiras variou entre as espécies florestais testadas e com a posição cerne 
interno e alburno, tendo os maiores valores para o cerne mediano e externo (Tabela 2), exceto para as madeiras de Tectona grandis e Caesalpinia echinata, com maiores densidades no alburno e cerne interno, respectivamente. As porcentagens de extrativos solúveis em etanol:tolueno foram maiores na posição externa do cerne de Acacia mangium, Eucalyptus cloeziana e Corymbia torelliana e na mediana do cerne de Casuarina equisetifolia, Tectona grandis e Caesalpinea echinata.

A perda de massa variou entre as espécies e posiçõesestudadas(Tabela2). Commenoresporcentagens para Corymbia torelliana e Caesalpinia echinata com média geral de I, 10 e 0,86\%, respectivamente. $O$ alburno da primeira madeira e o cerne interno da segunda foram os mais consumidos, respectivamente.

Casuarina equisetifolia foi a mais consumida, com média geral de 5,05\%, especialmente seu cerne interno, enquanto as demais madeiras tiveram perda intermediária de massa. As posições avaliadas das madeiras de Acacia mangium e Eucalyptus cloeziana não tiveram grandes variações de consumo pelos térmitas, com diferença máxima de I,80 e I,08\%, respectivamente. Enquanto a Tectona grandis teve o alburno mais consumido, com perda de massa de 2,59 vezes maior que a do cerne, que apresentou valor médio de 2,37\%. Isto está em conformidade com o obtido por Paes et al. (2007) e Motta et al. (20I3), que obtiveram maior perda de massa para o alburno da Tectona grandis, em relação às demais posições da madeira.

O período de sobrevivência de Nasutitermes corniger foi menor em contato com as madeiras de Corymbia torelliana e Caesalpinia echinata, 14,80 e 16,45 dias, respectivamente (Tabela 2). Por outro lado, os insetos foram mais longevos em contato com Tectona

Tabela 2 Densidade, extrativos, perda de massa, desgaste causado nos corpos de prova e tempo para a morte dos térmitas.

Table 2 Density, extractives, mass loss, waste caused on the test samples and time to death of termites.

\begin{tabular}{|c|c|c|c|c|c|c|}
\hline Espécie & Posição & $\begin{array}{l}\text { Densidade } \\
\left(\mathrm{g} \cdot \mathrm{cm}^{-3}\right)\end{array}$ & $\begin{array}{c}\text { Extrativos } \\
(\%)\end{array}$ & $\begin{array}{c}\text { Perda massa } \\
\qquad(\%)\end{array}$ & $\begin{array}{l}\text { Desgaste } \\
\text { (Notas) }\end{array}$ & $\begin{array}{l}\text { Tempo } \\
\text { (Dias) }\end{array}$ \\
\hline \multirow{4}{*}{$\begin{array}{l}\text { Acacia } \\
\text { mangium }\end{array}$} & Cerne interno & 0,95 & 8,01 & 2,97 & 9,20 & 20,60 \\
\hline & Cerne mediano & 1,02 & 6,51 & 2,32 & 9,76 & 20,60 \\
\hline & Cerne externo & 0,82 & 9,88 & 3,17 & 9,60 & 18,60 \\
\hline & Alburno & 0,71 & 5,92 & $\mathrm{I}, 37$ & 7,40 & 19,00 \\
\hline \multirow{4}{*}{$\begin{array}{l}\text { Casuarina } \\
\text { equisetifolia }\end{array}$} & Cerne interno & 0,37 & 7,14 & 9,99 & 5,08 & 21,40 \\
\hline & Cerne mediano & 0,49 & 10,47 & 1,92 & 7,40 & 16,80 \\
\hline & Cerne externo & 0,51 & 4,11 & 4,67 & 7,24 & 20,00 \\
\hline & Alburno & 0,49 & 1,33 & 3,57 & 7,92 & 14,40 \\
\hline \multirow{3}{*}{$\begin{array}{l}\text { Eucalyptus } \\
\text { cloeziana }\end{array}$} & Cerne interno & 0,57 & $4,4 I$ & 2,59 & 8,08 & 17,00 \\
\hline & Cerne mediano & 0,77 & 5,50 & 2,26 & 7,92 & 19,40 \\
\hline & Cerne externo & 0,89 & 6,27 & 1,68 & 8,60 & 18,20 \\
\hline \multirow{5}{*}{$\begin{array}{l}\text { Tectona } \\
\text { grandis }\end{array}$} & Alburno & 0,82 & 2,16 & 2,76 & 6,56 & 25,20 \\
\hline & Cerne interno & 0,60 & 3,49 & 1,10 & 8,00 & 22,20 \\
\hline & Cerne mediano & 0,62 & 6,38 & 3,24 & 6,52 & 23,60 \\
\hline & Cerne externo & 0,69 & 4,20 & 2,67 & 7,44 & 23,00 \\
\hline & Alburno & 0,72 & 1,61 & 6,16 & 5,08 & 28,00 \\
\hline \multirow{4}{*}{$\begin{array}{l}\text { Corymbia } \\
\text { torelliana }\end{array}$} & Cerne interno & 0,55 & 8,87 & 0,38 & 9,80 & 16,40 \\
\hline & Cerne mediano & 0,61 & 8,80 & $\mathrm{I}, 54$ & 9,76 & 14,60 \\
\hline & Cerne externo & 0,61 & 9,49 & 0,24 & 9,60 & 14,40 \\
\hline & Alburno & 0,49 & 5,31 & 2,25 & 8,00 & 13,80 \\
\hline \multirow{4}{*}{$\begin{array}{l}\text { Caesalpinia } \\
\text { echinata }\end{array}$} & Cerne interno & 0,92 & 9,57 & 1,00 & 9,92 & 13,20 \\
\hline & Cerne mediano & 0,84 & 10,15 & 0,98 & 9,96 & 16,20 \\
\hline & Cerne externo & 0,85 & 8,85 & 0,66 & 9,96 & 18,20 \\
\hline & Alburno & 0,80 & 6,25 & 0,81 & 9,12 & 18,20 \\
\hline Pinus sp. & Alburno & 0,53 & - & 4,43 & 7,49 & 15,25 \\
\hline
\end{tabular}


grandis, atingindo 28 dias na madeira de alburno. Para as demais espécies estudadas, não foram observadas grandes diferenças entre o tempo de sobrevivência dos térmitas, com médias variando de 18,15 (Acacia mangium) a 19,90 dias (Eucalyptus cloeziana).

A resistência natural, com base nas notas de desgaste (Tabela I) apresentou menores valores para as madeiras de Casuarina equisetifolia, Eucalyptus cloeziana e Tectona grandis, classificadas com ataque moderado, tendo médias de 6,91; 7,79; e 6,76, respectivamente. O cerne interno da Casuarina equisetifolia foi o mais consumido, com desgaste próximo ao ataque intenso. As madeiras de alburno de Eucalyptus cloeziana e Tectona grandis foram as mais consumidas, com ataques próximos ao moderado e intenso, respectivamente.

Por outro lado, as madeiras de Acacia mangium, Corymbia torelliana e Caesalpinea echinata foram as mais resistentes, com desgaste entre superficial e sadio. Apenas as madeira de alburno de Acacia mangium e Corymbia torelliana tiveram dano moderado.

A madeira de Pinus sp. utilizada como padrão de comparação (ASTM D - 3345, 2005) teve maior densidade que a madeira de Casuarina equisetifolia e semelhante a de Corymbia toreliana. A perda de massa dessa madeira foi próxima a da Casuarina equisetifolia, com desgaste semelhante a das madeiras de Casuarina equisetifolia e Tectona grandis e morte dos térmitas com valores intermediários aos das madeiras de Corymbia torelliana e Caesalpinea echinata.

Esperava-se que a madeira de Pinus sp. fosse a mais consumida pelos térmitas testados, em decorrência de sua baixa resistência a organismos xilófagos. Resultados semelhantes foram obtidos por Paes e Vital (2000), Paes et al. (2007), Rodrigues e Brito (20 I I). Paes et al. (2007) que atribuíram este fato à maior ocorrência deste gênero de térmitas em florestas de folhosas, tendo menor ocorrência em plantio de Pinus sp., gerando o menor hábito em consumir tal madeira. Supriana (1985) afirmou que térmitas são muito sensíveis às mudanças em suas dietas, não consumindo madeiras com as quais têm pouco contato.

Os valores de teor de extrativos e densidade da madeira (Tabela 2) indicaram correlação significativa entre o teor de extrativos em etanol:tolueno para a variável desgaste para as espécies Eucalyptus cloeziana, Corymbia torelliana e Caesalpinia echinata (Tabela 3). Para estas espécies o maior teor de extrativos correspondeu à maior nota de desgaste, significando um menor ataque à madeira.

Não houve correlação significativa entre o teor de extrativos e a perda de massa ao se avaliar as espécies estudadas. A correlação entre o teor de extrativos e a resistência natural da madeira de Tectona grandis a térmitas não foi significativa, segundo Lukmandaru (20I I), ao avaliar separadamente as posições de cerne e alburno, indicando que a resistência não está ligada à quantidade de extrativos, mas sim ao tipo de extrativo que seja tóxico aos térmitas.

Correlação significativa entre perda de massa e teor de extrativos para o cerne e alburno não foi observada por Paes et al. (20/3) ao estudarem a resistência de quatros espécies florestais a térmitas. $\bigcirc$ que corrobora o fato de que a resistência natural, na maioria das vezes, não ser atribuída ao conteúdo total de extrativos, mas sim à presença de compostos individuais que mesmo em pequenas quantidades são tóxicos a organismos xilófagos (THULASIDAS; BHAT, 2007).

$\mathrm{O}$ efeito dos extrativos foi significativo, quando se avaliaram as espécies em conjunto (entre espécies) para todas as variáveis avaliadas (Tabela 3). Isto indica que espécies com maior quantidade de extrativos em etanol:tolueno foram mais resistente ao cupins.

A densidade e as varáveis estudadas não apresentaram correlação significativa, exceto para Casuariana equisetifolia em que foi observada correlação significativa para a densidade e o desgaste provocado pelos cupins na madeira. Madeiras de nove espécies florestais não apresentaram relacionou significativa entre a densidade e a perda de massa causada por térmitas (PAES et al., 2003).

A densidade para as espécies em conjunto (entre espécies) correlacionou-se com a perda de massa e o desgaste causado pelos insetos. A correlação foi negativa para a perda de massa e positiva para o desgaste indicando que madeiras mais densas são menos consumidas pelos térmitas. Madeiras densas, geralmente possuem maiores teores de extrativos e, por isto, a resistência não estaria relacionada diretamente com a densidade e sim com a classe e quantidade de extrativos presentes na madeira (FINDLAY, 1985). Madeiras mais ricas em cinzas (compostos minerais e sílica) são menos consumidas pelo térmita Nasutitermes corniger em decorrência do desgaste causado às mandíbulas dos insetos (PAES et al., 20I3).

Houve diferença significativa para a interação espécie $x$ posição para todos os fatores testados (perda de massa, desgaste e tempo) (Tabela 2). Para as espécies Acacia mangium, Eucalyptus cloeziana e Caesalpinia echinata não houve diferença significativa entre as posições nas madeiras amostradas (Tabela 4), indicando 
Tabela 3 Correlações entre extrativos e densidade para perda de massa, desgaste e tempo para a morte dos cupins para cada espécie estudada.

Table 3 Correlations between extractives and density to mass loss, waste and time for the death of termites for each studied species.

\begin{tabular}{|c|c|c|c|c|c|c|}
\hline \multirow{3}{*}{ Espécie } & \multicolumn{6}{|c|}{ Correlações } \\
\hline & \multicolumn{3}{|c|}{ Extrativos (\%) } & \multicolumn{3}{|c|}{ Densidade $\left(\mathrm{g} \cdot \mathrm{cm}^{-3}\right)$} \\
\hline & $\begin{array}{c}\text { Perda Massa } \\
(\%)\end{array}$ & $\begin{array}{c}\text { Desgaste } \\
\text { (Nota) }\end{array}$ & $\begin{array}{c}\text { Tempo } \\
\text { (Dia) }\end{array}$ & $\begin{array}{c}\text { Perda } \\
\text { Massa (\%) }\end{array}$ & $\begin{array}{c}\text { Desgaste } \\
\text { (nota) }\end{array}$ & $\begin{array}{r}\text { Tempo } \\
\text { (Dia) }\end{array}$ \\
\hline Acacia mangium & $0,88^{\text {ns }}$ & $0,57^{\mathrm{ns}}$ & $-0,35^{\text {ns }}$ & $0,47^{\text {ns }}$ & $0,79^{\text {ns }}$ & $0,86^{\mathrm{ns}}$ \\
\hline Casuarina equisetifolia & $-0,02^{\text {ns }}$ & $-0,35^{\mathrm{ns}}$ & $0,3 I^{\mathrm{ns}}$ & $-0,89^{\text {ns }}$ & $0,94 *$ & $-0,58^{\text {ns }}$ \\
\hline Eucalyptus cloeziana & $-0,88^{\text {ns }}$ & $0,94 *$ & $-0,80^{\text {ns }}$ & $-0,55^{\mathrm{ns}}$ & $-0,06^{\mathrm{ns}}$ & $0,42^{\mathrm{ns}}$ \\
\hline Tectona grandis & $-0,48^{\text {ns }}$ & $0,39^{\text {ns }}$ & $-0,65^{\mathrm{ns}}$ & $0,79^{\text {ns }}$ & $-0,67^{\text {ns }}$ & $0,75^{\mathrm{ns}}$ \\
\hline Corymbia torelliana & $-0,85^{\mathrm{ns}}$ & $0,96 *$ & $0,52^{\mathrm{ns}}$ & $-0,57^{\text {ns }}$ & $0,83^{\text {ns }}$ & $0,12^{\text {ns }}$ \\
\hline Caesalpinia echinata & $0,47^{\text {ns }}$ & $0,95^{*}$ & $-0,59^{\text {ns }}$ & $0,46^{\mathrm{ns}}$ & $0,67^{\mathrm{ns}}$ & $-0,88^{\text {ns }}$ \\
\hline Entre Espécies & $-0,35^{*}$ & $0,62 * *$ & $-0,53$ *** & $-0,38 *$ & $0,48 * *$ & $0,14^{\mathrm{ns}}$ \\
\hline
\end{tabular}

** Significativo a I \% probabilidade; * significativo a 5\% probabilidade; ns não significativo pelo teste t de Student.

Tabela 4 Perda de massa, desgaste e tempo para a morte dos térmitas para as espécies florestais e posições na madeira.

Table 4 Mass loss, waste and time for the death of the termites for the tree species and wood positions.

\begin{tabular}{|c|c|c|c|c|}
\hline \multirow{2}{*}{ Espécie } & \multicolumn{4}{|c|}{ Perda de massa (\%) por posição na madeira } \\
\hline & Cerne interno & Cerne mediano & Cerne externo & Alburno \\
\hline Acacia mangium & $2,97 \mathrm{Ab}$ & $2,32 \mathrm{Aa}$ & $3,17 \mathrm{Aa}$ & $\mathrm{I}, 37 \mathrm{Ab}$ \\
\hline Casuarina equisetifolia & $9,99 \mathrm{Aa}$ & $\mathrm{I}, 9 \mathrm{I} \mathrm{Ca}$ & $4,67 \mathrm{Ba}$ & $3,57 \mathrm{Ba}$ \\
\hline Eucalyptus cloeziana & $2,59 \mathrm{Ab}$ & $2,26 \mathrm{Aa}$ & $\mathrm{I}, 68 \mathrm{Aa}$ & $2,75 \mathrm{Aa}$ \\
\hline Tectona grandis & $\mathrm{I}, 10 \mathrm{Bc}$ & $3,23 \mathrm{Ba}$ & $2,67 \mathrm{Ba}$ & $6,16 \mathrm{Aa}$ \\
\hline Corymbia torelliana & $0,38 \mathrm{Bc}$ & $\mathrm{I}, 54 \mathrm{Aa}$ & $0,23 \mathrm{Bb}$ & $2,24 \mathrm{Aa}$ \\
\hline Caesalpinia echinata & $\mathrm{I}, 00 \mathrm{Ac}$ & $0,98 \mathrm{Aa}$ & $0,66 \mathrm{Ab}$ & $0,8 \mathrm{IAb}$ \\
\hline \multirow{2}{*}{ Espécie } & \multicolumn{4}{|c|}{ Desgaste (nota) por posição na madeira } \\
\hline & Cerne interno & Cerne mediano & Cerne externo & Alburno \\
\hline Acacia mangium & $9,20 \mathrm{Aa}$ & $9,76 \mathrm{Aa}$ & $9,60 \mathrm{Aa}$ & $7,40 \mathrm{Ba}$ \\
\hline Casuarina equisetifolia & $5,08 \mathrm{Bc}$ & $7,40 \mathrm{Ab}$ & $7,24 \mathrm{Ab}$ & $7,92 \mathrm{Aa}$ \\
\hline Eucalyptus cloeziana & $8,08 \mathrm{Ab}$ & $7,92 \mathrm{Ab}$ & $8,60 \mathrm{Aa}$ & $6,56 \mathrm{Bb}$ \\
\hline Tectona grandis & $8,00 \mathrm{Ab}$ & $6,52 \mathrm{Bb}$ & $7,44 \mathrm{Ab}$ & $5,08 \mathrm{Cc}$ \\
\hline Corymbia torelliana & $9,80 \mathrm{Aa}$ & $9,76 \mathrm{Aa}$ & $9,60 \mathrm{Aa}$ & $8,00 \mathrm{Ba}$ \\
\hline Caesalpinia echinata & $9,92 \mathrm{Aa}$ & $9,96 \mathrm{Aa}$ & $9,96 \mathrm{Aa}$ & $9,12 \mathrm{Aa}$ \\
\hline \multirow{2}{*}{ Espécie } & \multicolumn{4}{|c|}{ Tempo (dias) por posição na madeira } \\
\hline & Cerne interno & Cerne mediano & Cerne externo & Alburno \\
\hline Acacia mangium & $20,60 \mathrm{Aa}$ & $20,60 \mathrm{Aa}$ & $18,60 \mathrm{Aa}$ & $19,00 \mathrm{Ab}$ \\
\hline Casuarina equisetifolia & $2 \mathrm{I}, 40 \mathrm{Aa}$ & $16,80 \mathrm{Bb}$ & $20,00 \mathrm{Aa}$ & $14,40 \mathrm{Bc}$ \\
\hline Eucalyptus cloeziana & $17,00 \mathrm{Bb}$ & $19,40 \mathrm{Ba}$ & $18,20 \mathrm{Ba}$ & $25,20 \mathrm{Aa}$ \\
\hline Tectona grandis & $22,20 \mathrm{Aa}$ & $23,60 \mathrm{Aa}$ & $23,00 \mathrm{Aa}$ & $28,00 \mathrm{Aa}$ \\
\hline Corymbia torelliana & $16,40 \mathrm{Ab}$ & $14,60 \mathrm{Ab}$ & I4,40 Aa & $13,80 \mathrm{Ac}$ \\
\hline Caesalpinia echinata & $13,20 \mathrm{Ab}$ & $16,20 \mathrm{Ab}$ & $18,20 \mathrm{Aa}$ & $18,20 \mathrm{Ab}$ \\
\hline
\end{tabular}

Médias seguidas da mesma letra, maiúscula na horizontal, ou minúscula na vertical, não diferem estatisticamente pelo teste de Scott-Knott $(p>0,05)$. 
que independentemente da posição testada a perda de massa não variou de forma significativa. No entanto, para as demais madeiras testadas a perda de massa foi afetada pala direção medula-casca.

A madeira mais resistente de Casuarina equisetifolia foi o cerne intermediário e a menos resistente o cerne interno. As resistências das madeiras de cerne externo e alburno apresentaram valores semelhantes.

A perda de massa de Tectona grandis foi maior no alburno. Corymbia Torelliana teve maior perda de massa também no alburno, porém não diferiu significativamente da madeira do cerne intermediário.

O cerne interno de Casuarina equisetifolia foi a madeira menos resistente; e o das madeiras de Tectona grandis, Corymbia torelliana e Caesalpinia echinata o mais resistente. As madeiras de Acacia mangium e Eucalyptus cloeziana tiveram resistência intermediária.

A resistência biológica da madeira do cerne intermediário foi semelhante entre as espécies, mas diferiu entre aquelas do cerne externo e alburno. $O$ cerne externo de Corymbia torelliana e Caesalpinia echinata tiveram maior resistência que as demais, enquanto o alburno de Acacia mangium e de Caesalpinia echinata foi mais resistente entre as espécies estudadas.

O desgaste do alburno de Acacia mangium, Eucalyptus cloeziana e Corymbia torelliana tiveram as menores notas, indicando um maior consumo pelos térmitas que madeiras das posições do cerne. A madeira de Casuarina equisetifolia apresentou menor resistência para o cerne interno e a Caesalpinia echinata não apresentou diferenças entre as posições testadas.

O cerne interno das madeiras de Acacia mangium, Corymbia torelliana e Caesalpinia echinata foi mais resistente e da Casuarina equisetifolia o menos resistente, tendo as madeiras Eucalyptus cloeziana e de Tectona grandis, resistência intermediária.

A resistência das madeiras de cerne intermediário e externo teve comportamento semelhante, com a Acacia mangium, Corymbia torelliana e Caesalpinia echinata as mais resistentes, exceto para Eucalyptus cloeziana cujos cernes interno e externo tiveram resistências diferentes.

A madeira de alburno de Acacia mangium, Casuarina equisetifolia, Corymbia torelliana e Caesalpinia echinata foram as mais resistentes, a de Tectona grandis foi a menos resistente e a de Eucalyptus cloeziana teve resistência intermediária.

A mortalidade dos térmitas foi semelhante em madeira das diversas posições de Acacia mangium, Tectona grandis, Corymbia torelliana e Caesalpinia echinata, enquanto isto foi maior na madeira de alburno de
Eucalyptus cloeziana que nas demais posições avaliadas. O tempo de sobrevivência dos térmitas foi maior nos cernes interno e externo, que no intermediário e alburno de Casuarina equisetifolia.

Variações na resistência biológica das madeiras dos cernes interno, intermediário e externo tem sido relatadas (PAES; VITAL, 2000; PAES et al., 2003; AMUSANT et al., 2004; BHAT et al., 2005; PAES et al., 2007; ALENCAR et al.; 20I I) e são causadas pelos diferentes tipos e quantidades de extrativos existentes, sem um padrão de concentração entre as posições do cerne para as diversas espécies florestais ou posições longitudinais no tronco (FINDLAY, 1985). Térmitas morrem mais rapidamente em contato com madeiras com maiores teores de extrativos (PAES et al.; 2003; 2007), mas isto não foi observado nesta pesquisa, provavelmente por causa do tipo de substâncias extraídas em etanol:tolueno, as quais não são muito tóxicas a térmitas da espécie Nasutitermes corniger.

\section{CONCLUSÕES}

A resistência natural da madeira, dentre as posições medula-casca, para as espécies estudadas, não está correlacionada com a densidade e teor de extrativos das mesmas. No entanto, entre as madeiras, aquelas com maior densidade e teor de extrativos são mais resistentes.

A resistência biológica das madeiras varia entre as posições no sentido medula-casca, mas sem um mesmo comportamento entre as posições para todas as espécies estudadas.

As madeiras com maior resistência biológica ao térmita Nasutitermes corniger (menores perda de massa, desgaste e tempo de sobrevivência dos insetos) são Corymbia torelliana e Caesalpinia echinata e a de menor resistência a Casuarina equisetifolia.

\section{REFERÊNCIAS}

ALENCAR, F.H.H.; PAES, J. B.; BAKKE, O. A.; SILVA, G.S. Resistência natural da madeira de sabiá (Mimosa caesalpiniifolia Benth.) a cupins subterrâneos. Revista Caatinga, Mossoró, v. 24, n. I, p. 57-64, 201 I.

ALMEIDA, F. D. Propagação vegetativa de Eucalyptus cloeziana F. Muell. por estaquia e miniestaquia. 2006. 86 f. Dissertação (Mestrado em Ciência Florestal) Universidade Federal de Viçosa, Viçosa, 2006.

AMERICAN SOCIETY FOR TESTING AND MATERIALS. ASTM D - I 107: standard test method for ethanoltoluene solubility of wood. Annual Book of ASTM Standard, Philadelphia, 2005. 2p. 
AMERICAN SOCIETY FOR TESTING AND MATERIALS. ASTM D - 14I3: standard test method for wood preservatives by laboratory soil-block cultures. Annual Book of ASTM Standard, Philadelphia, 2005. 7p.

AMERICAN SOCIETY FOR TESTING AND MATERIALS. ASTM D - 3345: standard method for laboratory evaluation of the wood and other cellulosic materials for resistance to termite. Annual Book of ASTM Standard, Philadelphia, 2005. 3p.

AMERICANSOCIETYFORTESTINGAND MATERIALS. ASTMD 2017: standard test method for accelerated laboratory test of natural decay resistance of wood. Annual Book of ASTM Standards, Philadelphia, 2005. 5p.

AMUSANT, N.; BEAUCHENE, J.; FOURNIER, M.; JANIN, G.; THEVENON, M. Decay resistance in Dicorynia guianensis Amsh: analysis of inter-tree and intra-tree variability and relations with wood colour. Annals of Forest Science, Les Ulis, v. 6I, n. 4, p. 373-380, 2004.

ANGYALOSSY, V.; AMONO, E.; ALVES, E. S. Madeiras utilizadas na fabricação de arcos para instrumentos de corda: aspectos anatômicos. Acta Botanica Brasilica, São Paulo, v. 19, n. 4, p. 819-834, 2005.

BALIEIRO, F. C.; DIAS, L. E.; FRANCO, A. A.; CAMPELLO, E.F.C.; FARIA, S.M. Acúmulo de nutrientes na parte aérea, na serapilheira acumulada sobre o solo e decomposição de filódios de Acacia mangium Willd. Ciência Florestal, Santa Maria, v. I4, n. I, p. 59-65, 2004

BHAT, K. M.; THULASIDAS, P. K.; MARIA FLORENCE, E. J.; JAYARAMAN K. Wood durability of home-garden teak against brown-rot and white-rot fungi. Trees, Vancouver, v. 19, n. 6, p. 654-660, 2005.

FINDLAY, W.P.K. Preservative substances: In: FINDLAY, W.P.K. (Ed.) Preservation of timber in the tropics. Dordrecht: Martinus Nijhoff/Dr. W. Junk Publishrs, 1985. p. 59-74.

GONÇALVES, F. G.; OLIVEIRA, J. T. S. Resistência ao ataque de cupim-de-madeira seca (Cryptotermes brevis) em seis espécies florestais. Cerne, Lavras, v. I2, n. I, p. 80-83, 2006.

HORNBURG, K. F.; ELEOTÉRIO, J. R.; BAGATTOLI, T. R.; NICOLETTI, A. L. Qualidade das toras e da madeira serrada de seis espécies de eucalipto cultivadas no litoral de Santa Catarina. Scientia Forestal, Piracicaba, v. 40, n. 96, p. 463-47I, 2012.

LUKMANDARU, G. Variability in the natural termite resistance of plantation teak wood and its relations with wood extractive content and color properties. Journal of Forestry Research, Harbin, v. 8, n. I, p.|7-3|, 201।.
MARQUES, S. S.; OLIVEIRA, J. T. S., PAES, J. B.; ALVES, E. S.; SILVA, A. G. S.; FIEDLER, N. C. Estudo comparativo da massa específica aparente e retratibilidade da madeira de pau-brasil (Caesalpinia echinata Lam.) nativa e de reflorestamento. Revista Árvore, Viçosa, v.36, n.2, p.373-380, 2012.

MOTTA, J. P.; OLIVEIRA, J. T. S.; PAES, J. B.; ALVES, R. C.; DAMBROZ, G. B. V. Resistência natural da madeira de Tectona grandis em ensaio de laboratório. Ciência Rural, Santa Maria, v. 43, n. 8, p. I393-1398, 2013.

PAES, J. B.; MELO, R. R.; LIMA, C. R. Resistência natural de sete madeiras a fungos e cupins xilófagos em condições de laboratório. Cerne, Lavras, v. 13, n. 2, p. 160-169, 2007.

PAES, J. B.; VITAL, B. R. Resistência natural da madeira de cinco espécies de eucalipto a cupins subterrâneos, em testes de laboratório. Revista Árvore, Viçosa, v. 24, n. I, p. 97-104, 2000.

PAES, J. B.; MEDEIROS NETO, P.N.; LIMA, C. R.; FREITAS, M. F.; DINIZ, C. E. F. Efeitos dos extrativos e cinzas na resistência natural de quatro madeiras a cupins xilófagos. Cerne, Lavras, v. 19, n. 3, p. 399-405, 2013.

PAES, J. B.; MORAIS, V. M.; FARIAS SOBRINHO, D. W.; BAKKE, O. A. Resistência natural de nove madeiras do semi-árido brasileiro a cupins subterrâneos. Cerne, Lavras, v. 9, n. I, p. 36-47, 2003.

PAES, J. B.; MORAIS, V. M.; LIMA, C. R.; SANTOS, G. J. C. Resistência natural de nove madeiras do semiárido brasileiro a fungos xilófagos em simulares de campo. Revista Árvore, Viçosa, v. 33, p. 5 I I-520, 2009.

PAES, J. B.; SOUZA, A. D.; LIMA, C. R.; MEDERIOS NETO, P. N. Eficiência dos óleos de nim e mamona contra cupins xilófagos em ensaio de alimentação forçada. Cerne, Lavras, v. 16, p. I05-113, 2010.

PANSHIN, A.J.; DE ZEEUW, C. Textbook of wood technology. 4 ed. New York: 1980. 722p.

RODRIGUES, R.B.; BRITO, E.O. Resistência natural de Eucalyptus urophylla e Corymbia citriodora a Coptotermes gestroi (Isoptera; Rhinotermitidae) em laboratório. Floresta e Ambiente, Seropédica, v. 18, n. I, p. 9-I5, 20 II.

RUDI, D.; BHAT, I. U. H.; ABDUL KHALIL, H.P.S., NAIF, A.; HERMAWAN, D. Evaluation of antitermitic activity of different extracts obtained from Indonesian teakwood (Tectona grandis L.f). BioResources, Raleigh, v. 7, n. 2, p. |452-|46I, 2012.

SILVA, J. C.; LOPEZ, A. G. C.; OLIVEIRA, J. T. S. Influência da idade na resistência natural da madeira de Eucalyptus grandis Hill ex. Maiden ao ataque de cupim de madeira seca (Cryptotermes brevis). Revista Árvore, Viçosa, v. 28, n.4, p. 583-588, 2004. 
SILVA, L. M.; HASSE, I.; CADORIN, D. A.; OLIVEIRA, K. A.; OLIVEIRA, F. A. C.; BETT, C. F. Inventário da arborização em duas vias de Mariópolis/PR. Revista da Sociedade Brasileira de Arborização Urbana, Piracicaba, v.3, n. I, p. 36-53, 2008.

STEEL, R. G. D.; TORRIE, J. H. Principles and procedures of statistic: a biometrical approach. 2.ed. New York: Mc Graw Hill, 1980. 633p.

SUPRIANA, N. Notes on the resistance of tropical wood against termites. Stockholm: The International Research Group on Wood Preservation, 1985. 9 p. (IRG/WP, I249).
THULASIDAS, P. K.; BHAT, K. M. Chemical extractive compounds determining the brown-rot decay resistance of teak wood. Holz als Roh- und Werkstoff, Berlin, v. 65, n. 2, p. I2I-I24, 2007.

TREVISAN, H. Degradação natural de toras e sua influência nas propriedades físicas e mecânicas da madeira de cinco espécies florestais. 2006. 56 f. Dissertação (Mestrado em Ciências Florestais e Ambientais) - Universidade Federal Rural do Rio de Janeiro, Seropédica, 2006. 
